\title{
Single Incision Multiport Laparoscopic Surgery (SILS) with Conventional Instruments: A Single Centre Experience
}

\author{
Kulkarni Akshay, Rege Sameer \\ Department of Surgery, Seth G S Medical College \& KEM Hospital, Parel, Mumbai.12
}

\begin{abstract}
Background: Single incision laparoscopic surgery (SILS) is an evolving field. Specially designed ports required for SILS are expensive, making this technique unaffordable to many. To overcome this drawback, the use of conventional affordable instruments for SILS was encouraged. We present our case series of SILS with conventional instruments in a tertiary care centre.

Methods: This is a single centre pilot study of 30 consecutive cases operated by SILS using conventional laparoscopic instruments. Outcomes measured were operative time, post-operative pain by Visual analogue scale (VAS), resumption of oral diet, duration of stay in the hospital, wound cosmesis and complications.

Results: Thirty patients (21 females and nine males; mean age $35.05 \pm 12.06$ years) were operated by SILS procedure. Twenty single incision laparoscopic Cholecystectomies(SILC), seven single incision laparoscopic appendectomies (SILA) and three single incision diagnostic laparoscopies(SIDL) were done with average operative time of 92 minutes, 47 minutes and 53 minutes respectively. All surgeries were performed successfully without conversion to conventional laparoscopy or open method. Majority patients were given oral feeds and discharged on the second postoperative day without any complications. Wound cosmesis was satisfactory with an inconspicuous scar in all patients.

Conclusion: Our initial experience of using conventional laparoscopic instruments for SILS demonstrates its feasibility and utility especially in resource challenged settings. The advantage of SILS is its reduced need of analgesics and better wound cosmesis, without significant post-operative complications.
\end{abstract}

Keywords: Single-incision multiport laparoscopic surgery, appendectomy, cholecystectomy

\section{Introduction}

The laparoscopic techniques have progressed from conventional multiple incisions to reduced port laparoscopic surgery; adding the benefit of reduced pain and fewer scars. ${ }^{[1]}$ In last few decades it has further progressed to single incision laparoscopic surgery with sophisticated articulating instruments. Single incision laparoscopic surgery (SILS) is a type of laparoscopic surgery which involves use of a single incision of $1.5-2 \mathrm{~cm}$ (usually infra-umbilical) through which all the required ports or a single specially designed port is inserted. ${ }^{[1]}$

SILS is a rapidly evolving field of minimally invasive surgery and offers better wound cosmesis without significant increase in operating time or post-operative complications ${ }^{[1]}$. The final operative site scar at the umbilicus is cosmetically acceptable and hardly visible after a month. SILS is currently adapted to different surgical procedures such as appendectomy, colectomy, nephrectomy, splenectomy, bariatric surgeries and many others. ${ }^{[2]}$.

Major issue with the development of SILS is the availability of ergonomic, articulating and flexible roticulating special instruments and modern expensive ports. ${ }^{[3]}$ In a resource constrained setting this can be a major hindrance to its widespread acceptance and utility. To overcome this disadvantage a need was felt to use conventional rather than the special instruments for SILS thus reducing economic burden and increasing the acceptance. Our study of 30 cases of SILS with conventional instruments is based on this felt need of resource challenged settings.

\section{Materials and Methods}

This was a pilot study of 30 cases of SILS with the use of conventional laparoscopy instruments performed in a single tertiary care centre. All the patients underwent surgery by a single surgeon, experienced in laparoscopic surgery. Patient demographic data was recorded as per the database under an institutional review board. The cases included were patients of both sexes and were above 18 years of age. Pregnant patients and children were excluded. Patients were informed about the technique and written informed consent was obtained under the guidance of Institutional Ethics committee. All cases were studied in terms of clinical presentation, radiological investigations, operative findings and postoperative course. The conventional laparoscopic instruments available at the centre were used and no special ports or instruments were used for these surgeries.

Three types of surgeries included in series were single incision multiport laparoscopic cholecystectomy (SILC), single incision multiport diagnostic laparoscopy (SIDL) and single incision multiport appendectomy (SILA). 


\section{Single incision multiport laparoscopic cholecystectomy}

\section{Operative methods}

The patients were positioned on the operating table in a reverse Trendelenburg, right side up position. A single curved, infra-umbilical, $2.0 \mathrm{~cm}$ incision was made. After exposing the fascia, a $10-\mathrm{mm}$ trocar was placed through an open approach and into the peritoneum and insufflated up to $12 \mathrm{mmHg}$ with CO2.The abdominal cavity was explored with a 10-mm laparoscope, then two $5 \mathrm{~mm}$ ports were placed through the same umbilical incision but through separate fascial incisions. Both the ports were placed slightly laterally from the laparoscope port. For optimal exposure and appropriate traction two percutaneous sutures were passed into abdominal cavity. First through fundus of gall bladder and second through Hartman's pouch (Fig. 1). Dissection was performed as a normal retrograde cholecystectomy.

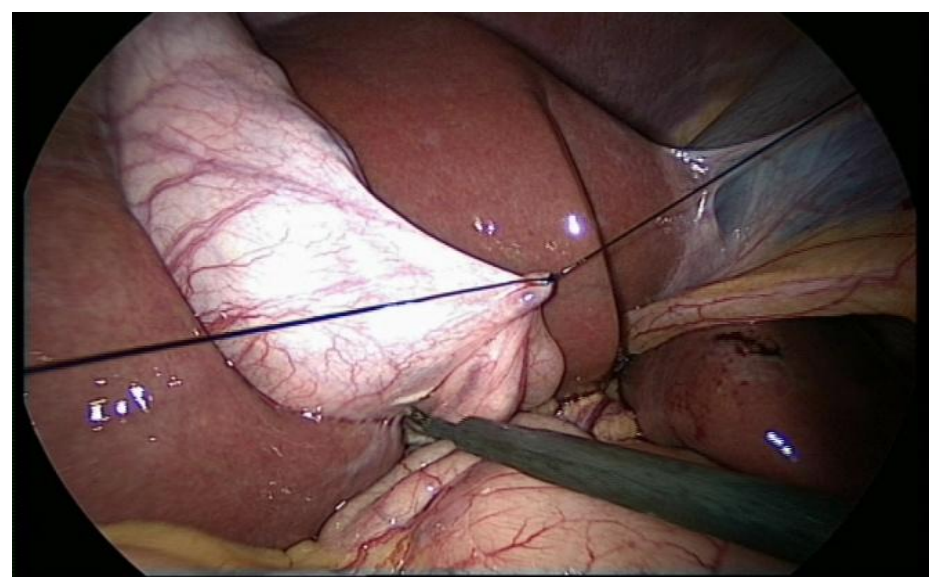

Fig.1 - Per-abdominal suture passed through Hartman’s pouch

\section{Single incision multiport laparoscopic appendectomy}

A single infra-umbilical curved skin incision measuring $1.5 \mathrm{~cm}$ was given. Two separate fascial incisions for two ports were taken (Fig.2). Entire peritoneal cavity was visualized to confirm the diagnosis and note the position of appendix. Appendix was caught with Babcock forceps and meso-appendix was cauterized using bipolar diathermy. Base of the appendix was ligated and cut between two proximal and one distal ligature and appendix was retrieved.

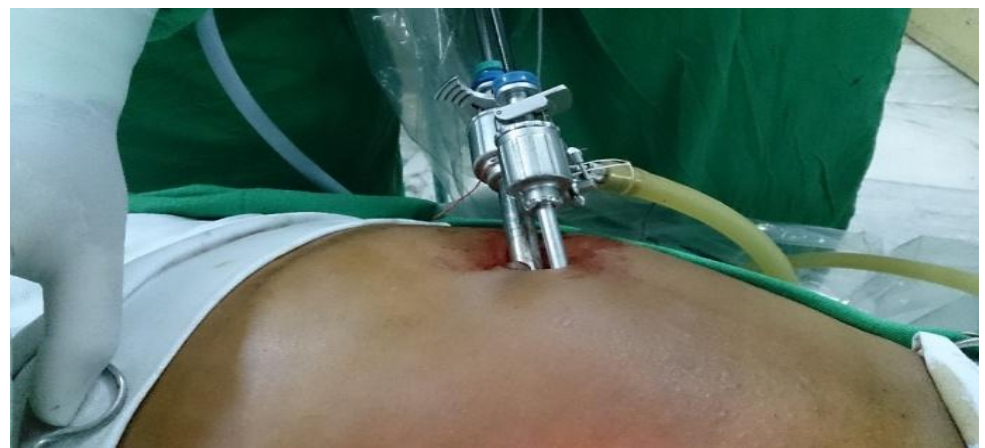

Fig.2 - Port Placement during the SILS

\section{Single incision multiport Diagnostic laparoscopy}

A single infra-umbilical curved skin incision measuring $1.5 \mathrm{~cm}$ was given. Two separate fascial incisions for two ports were taken. Entire peritoneal cavity was visualized to confirm the diagnosis.

\section{Results}

Thirty patients underwent SILS procedure. Twenty patients underwent single incision multiport laparoscopic cholecystectomy (SILC). Seven patients with recurrent appendicitis underwent single incision multiport laparoscopic appendectomy (SILA) and three patients underwent single incision multiport diagnostic laparoscopy (SIDL) for undiagnosed chronic abdominal pain. The average age was $35.05 \pm 12.06$ years, and there were nine males and 21 females. Three umbilical ports were utilized in SILC cases while the other cases required only two umbilical ports. 
There were no conversions to a conventional laparoscopic approach or to open surgeries. The mean operative time was $82 \pm 33.6$ min with minimal blood loss. Most patients were given oral feeds and discharged on the second postoperative day. The use of analgesics and pain scores (VAS scale) were similar to our experience with conventional laparoscopic surgeries. Post procedure patients reported a mean VAS score of two during the hospital stay. None of the demographic or clinical features affected the operative time or Mean VAS score or hospital stay. The postoperative period was uneventful in all patients. No postoperative complications (seroma, surgical site infection) and no trocar-site hernia were observed at 6-month follow-up period after surgery. Surgical cosmesis was good resulting in an inconspicuous scar (Fig.3).

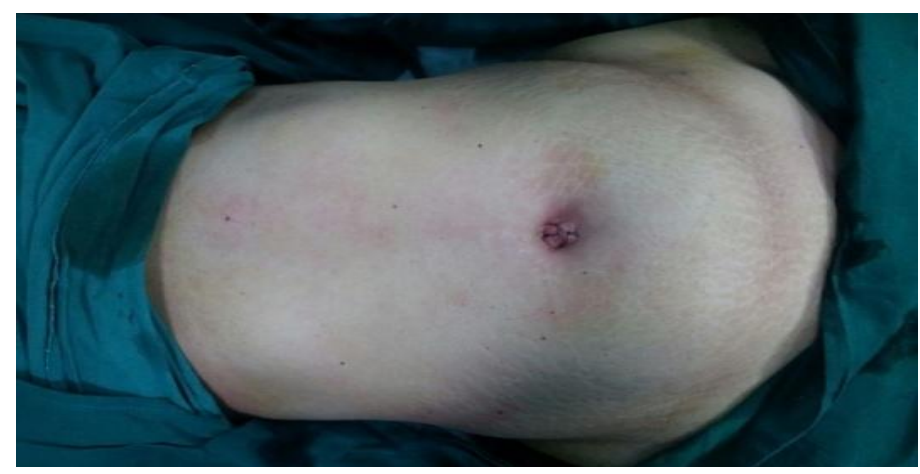

Fig.3 - Post operative view of the incision

\section{Discussion}

Laparoscopy has emerged as a strong contender in the field of general surgery since 1985 since the pioneering Cholecystectomy done by German surgeon Erich Muhe. ${ }^{[4]}$ With the definite evidence of the superiority of laparoscopy over conventional open surgeries, further advances in this field were sought. Progress in the field of laparoscopy initially involved advancements in light sources, sophisticated instruments and later centred on to lesser number of ports and wound cosmesis. ${ }^{[3]}$

In 1992 Pelosi and Pelosi performed first single incision laparoscopic appendectomy ${ }^{[5]}$. In 1997 , Navarra et al. first described one-incision laparoscopic cholecystectomy ${ }^{[6]}$. In 1999, Piskun and Rajpal performed SILS by inserting two trocars through the umbilical incision and putting additional stay sutures to retract and stabilize the gallbladder. ${ }^{[7]}$.

SILS is a rapidly evolving field of minimally invasive surgery and offers better wound cosmesis without significant increase in operating time or post-operative complications ${ }^{[1]}$. The final operative site scar at the umbilicus is cosmetically acceptable and hardly visible after a month. Two different methods have been described for trocar access to perform SILC.

1 Trans-umbilical single-port access (SPA) with following ports

A. Specially designed commercially available ports e.g., SILS ${ }^{\text {TM }}$ port or R-Port ${ }^{[8]}$

B. Indigenous Glove port though which multiple trocars can be passed ${ }^{[9]}$.

2 Multiple fascial punctures through one trans-umbilical skin incision for multiple ports. The use of additional transabdominal retraction sutures to retract the gallbladder was also found useful ${ }^{[10]}$.

C. Convectional trocars through separate facial incisions

Numerous advantages of SILS have been proposed, such as cosmesis ('scar-less' surgeries) reduced pain or need of analgesia, and ability to convert to standard four-port laparoscopic surgery when needed. ${ }^{[3]}$ SILS being a type of laparoscopic surgery, the disadvantages inherent to laparoscopic technique like 2dimensional view, loss of tactile/haptic sensation, semi-paradoxical hand motions and longer learning curve are observed. ${ }^{[3]}$ Disadvantage unique to SILS is loss of concept of triangulation. Triangulation forms the central basis of laparoscopic skills. The new parallel instrument positioning in SILS causes closely spaced instruments, both outside and inside the abdomen, hand clashing or chopstick effect; resulting in increased duration of learning curve. ${ }^{[3]}$ Newer ports, 'in line' telescopes, new generation of articulating instruments providing pseudo or cross triangulation have overcome these limitations ${ }^{[1]}$. However these sophisticated instruments add to the cost of surgery and hence practical utility and benefits of SILS remain out of reach for patients in resource poor setting. This led to the concept of using conventional affordable laparoscopic instruments to perform SILS. Few initial reports have been encouraging however the concrete data regarding this concept remains scarce. ${ }^{[1]}$ The results of our study are similar as those found in studies by Uday S.K et al, Sabuncuoglu et al and Sinha et al utilization of conventional laparoscopic instruments for SILS significantly decreased the costs without increasing the conversion rates or post-operative complications. ${ }^{[9,10,11]}$ Utilisation of conventional ports and instruments would considerably reduce the cost and definitely ensure development of SILS as a standard of care even in resource challenged settings. 
Almost all reported studies including present study have shown longer operating times. ${ }^{[3,10]}$ Our operating time improved after the learning curve and experience, but was always significantly more than that for conventional laparoscopy. The major reason is an inherent learning curve of any newer laparoscopic technique which was also observed when there was a transition from open to conventional laparoscopy. ${ }^{[12]}$. The longer operating times could also be due to use of conventional rigid laparoscopic instruments creating an ergonomically challenged operating field.

In our series, there were no instances of port site infections or hematoma in the immediate postoperative period as against a recent meta-analysis which quotes infection and hematoma as $2.1 \%$. ${ }^{[13]}$ In our study none of the patients developed wound complications or port site hernias on follow up of 6 months and all patients had good wound cosmesis. The results of study by Sabuncuoglu et al were also similar. ${ }^{[10]}$ In our study the mean VAS score for postoperative pain was about two; which is similar to conventional laparoscopy from our experience. However literature still remains unclear with varying reports of SILS resulting in lesser pain, ${ }^{[14]}$ more pain ${ }^{[15]}$ or no difference ${ }^{[16]}$ as compared with conventional laparoscopy. There are some limitations to our study like small sample size and lack of randomization and comparison with conventional laparoscopy. Larger randomized control trials are required for definite conclusions about efficacy and advantage of SILS with conventional instruments.

\section{Conclusion}

Our initial experience of using conventional laparoscopic instruments for SILS demonstrates its feasibility and utility especially in resource challenged settings with lack of modern specialised articulating instruments. The advantages of SILS are its reduced need of analgesics, excellent wound cosmesis, without significant post-operative complications.

\section{References}

[1]. Rao. P, Rao.P, and Bhagwat. S. Single-incision laparoscopic surgery - current status and controversies. J Minim Access Surg. 2011 Jan-Mar; 7(1): 6-16.

[2]. Ahmed K, Wang TT, Patel VM, Nagpal K, Clark J, Ali M, et al. The role of single-incision laparoscopic surgery in abdominal and pelvic surgery: a systematic review. Surg Endosc 2011; 25(2): 378-96

[3]. Khorgami Zh, Shoar S, Shoar N, Shakoor D, Mahdavian Sh, Nasiri Sh, Soroush A, Aminian A. Single Incision Laparoscopic Surgery: Review of Pros and Cons. Acad J Surg, 2014; 1(1): 25-32.

[4]. Grzegorz S. Litynski. Erich Mühe and the Rejection of Laparoscopic Cholecystectomy (1985): A Surgeon Ahead of His Time. JSLS. 1998 Oct-Dec; 2(4): 341-346.

[5]. Pelosi MA, Pelosi MA 3rd. Laparoscopic appendectomy using a single umbilical puncture (minilaparoscopy). J Reprod Med 1992; 37: 588-594

[6]. Navarra G, Pozza E, Occhionorelli S, Carcoforo P, Doninin I. One-wound laparoscopic cholecystectomy. Br J Surg. 1997;84:695

[7]. Piskun G, Rajpal S. Transumbilical laparoscopic cholecystectomy utilizes no incisions outside the umbilicus. J Laparoendosc Adv Surg Tech. 1999;9:361

[8]. Prashanth P. Rao, 1 Sonali M. Bhagwat,1 Abhay Rane,2 and Pradeep P. Rao The feasibility of single port laparoscopic cholecystectomy: a pilot study of 20 cases. HPB (Oxford). 2008 Oct 1; 10(5): 336-340.

[9]. Uday SK, Bhargav P. SILACIG: A novel technique of single-incision laparoscopic appendicectomy based on institutional experience of 29 cases. J Min Access Surg 2013; 9:76

[10]. Sabuncuoglu, M.Z., Sabuncuoglu, A., Sozen, I., Tozlu, G., Benzin, M.F. and Cetin, R. (2014) Single-Incision Laparoscopic Cholecystectomy with Conventional Instruments: A Surgeon's Initial Experience. Surgical Science, 5,299-305

[11]. Sinha. R, Yadav. A. Transumbilical single incision laparoscopic cholecystectomy with conventional instruments: A continuing study. Journal of Minimal Access Surgery. October-December 2014; Volume 10: Issue 4.

[12]. Joseph M, Phillips M, Rupp CC. Single-incision laparoscopic cholecystectomy: a combined analysis of resident and attending learning curves at a single institution. Am Surg 2012; 78(1): 119-24

[13]. Antoniou SA, Pointner R, Granderath FA. Single incision laparoscopic cholecystectomy: A systematic review. Surg Endosc 2011;25:367-77.

[14]. Bucher P, Pugin F, Buchs NC, Ostermann S, Morel P. Randomized clinical trial of laparoendoscopic single-site versus conventional laparoscopic cholecystectomy. Br J Surg 2011;98:1695-702 .

[15]. Lirici MM, Califano AD, Angelini P, Corcione F. Laparo-endoscopic single site cholecystectomy versus standard laparoscopic cholecystectomy: Results of a pilot randomized trial. Am J Surg 2011;202:45-52.

[16]. Ma J, Cassera MA, Spaun GO, Hammill CW, Hansen PD, Aliabadi-Wahle S. Randomized controlled trial comparing single-port laparoscopic cholecystectomy and four-port laparoscopic cholecystectomy. Ann Surg 2011;254:22-7 